\title{
A perception experiment with time-critical graphics animation on the World-Wide Web
}

\author{
HEIKO HECHT, MARKUS OESKER, ANDREAS KAISER, \\ HAKAN CIVELEK, and THOMAS STECKER \\ Universität Bielefeld, Bielefeld, Germany
}

\begin{abstract}
The World-Wide Web offers a potentially interesting tool to collect data from a large and heterogeneous audience. While questionnaires have become rather common on the Internet, its potential reaches far beyond text processing. In principle, it is possible not only to perform interactive, dynamic experiments on the Web, but also to include graphical animation and time-critical responses, such as reaction times. We implemented a visual motion extrapolation task on the Web using the programming language Java, which can be interpreted by standard Web browsers such as Netscape or Internet Explorer. The data collected with this method turned out to be reliable and differed little from data obtained in a controlled laboratory setting, with the exception of conditions with fixation instruction. Thus, the Web can, generally speaking, be used for data collection of large sample sizes. The strengths and weaknesses of dynamic visual simulation experiments on the Internet are discussed.
\end{abstract}

The Internet in general and the World-Wide Web (WWW) in particular have by now been proven to be a valuable tool for the behavioral sciences. Contributions have been made in the domains of psychology education (e.g., Bailey \& Cotlar, 1994; Trapp, Hammond, \& Bray, 1996), location of information sources (Kelley-Milburn \& Milburn, 1995), and questionnaire-based data collection (Hewson, Laurent, \& Vogel, 1996). In all of these domains, the advantages of the Internet have outweighed its disadvantages, and consequently the WWW has become a tool of choice in many situations. However, to this date, the possibilities of dynamic experimentation that have emerged with the developments of Java have hardly been explored. Java (see, e.g., Flanagan, 1996; Kent \& Kent, 1996) is an object-oriented programming language that is network capable; that is, it can be used by a variety of Web browsers. This paper reports our initial experiences with a dynamic psychological experiment that was implemented using Java. The quality of the visual animation as well as the data collection posed many problems, which could be overcome in most cases. Thus, dynamic experimentation on the Internet is not only feasible, but also promising for experimental situations in which a large international subject pool is needed.

We thank Darrell Butler and two anonymous reviewers for their insightful comments, which greatly improved this paper. We thank Bernhard Jung and Ipke Wachsmuth for their excellent support and for maintaining the Web server at the TechFak of the University of Bielefeld. Ulf Reips advertised the experiment in the Experimental Psychology Lab. Finally, thanks go to the volunteers around the world who participated in the experiment. Correspondence should be addressed to $\mathrm{H}$. Hecht, ZiF/Universität Bielefeld, Wellenberg 1,33615 Bielefeld, Germany (email: hecht@hrz.uni-bielefeld.de).

\section{Present Use and Potential of the WWW}

The potential capabilities of Java-based Internet experiments are enormous: An almost unlimited subject pool across geographical and cultural boundaries can be accessed, researchers who do not have on-site subject pools can perform experiments, the self-selection of participants could shift from undergraduate students enrolled in introductory psychology classes to computer literates of any age and profession, and, probably most importantly, experiments that require a very large number of observations become feasible. For instance, for a particular visual illusion that can be studied only with observers who have never seen it before, the subject supplies of psychology classes are quickly exhausted in traditional experimentation, whereas the Internet can draw on much larger numbers. Thus far, Java has mostly been used for unidirectional demonstrations. Obviously, these advantages of Internet experiments are accompanied by some sacrifices concerning the amount of control over the experimental setup and the monitoring of subjects.

Thus far, the graphics tools of the Internet have been used in the realm of perceptual psychology mostly to provide examples of visual illusions and other visual effects (for a starting point, see http://www.visionscience.com/ visionscience.html), including some interactive demonstrations (e.g., http://www.illusionworks.com/html/). However, to our knowledge the Web has not yet been explored for dynamic experimentation that involves animated graphics. A step in this direction has been made in the acoustic domain (Welch \& Krantz, 1996). The next paragraphs briefly describe the potential to extend the use of hypertext documents to dynamic visual animation.

With the creation of the WWW, millions of computers have been connected through the use of a standardized 


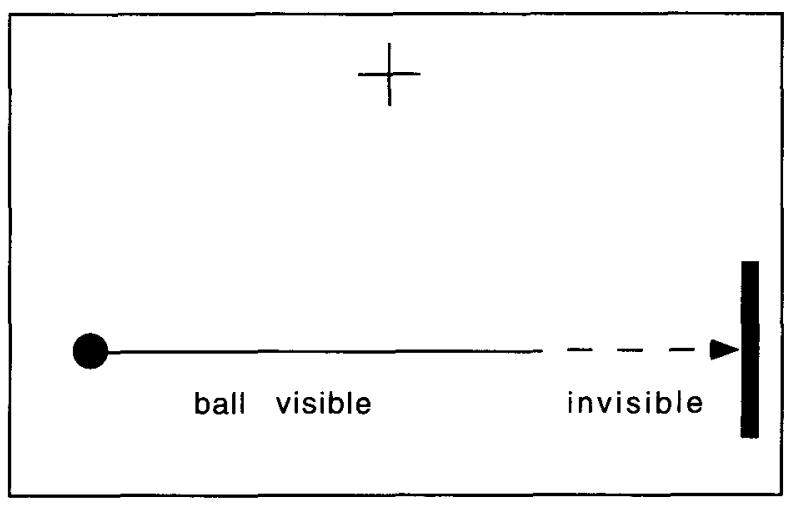

Figure 1. The ball appeared close to the left (or center bottom) of the screen and moved to the right (upward) as indicated. The target line represents the rightmost position that was used.

communication protocol, the so-called hypertext-transferprotocol (http). This protocol standardized data exchange of picture and sound files and other resources, such as text formatting, running of local helper programs, or the uniform address coding (uniform resource locator, or URL). The latter refers not only to a particular computer somewhere in the world, but also to a document residing on the user's machine.

Documents that are written in the appropriate hypertext format (hypertext markup language, or HTML) can be interpreted by various Web browsers, one of which has to be resident on the user's machine. Well-known features such as hotlinks establish connections between documents that can be spread out over an arbitrary number of computers located anywhere in the world. The latest feature of Web browsers such as Netscape or Explorer is their ability to interpret object code provided by the host through the Internet link. These applets, run on the local machine, are written in Java, a programming language especially designed for use with Internet browsers that has adopted many features of $\mathrm{C}^{++}$. We have programmed the present experimental displays using Java.

\section{Timed Event Handling With Java Applets}

Java is an object-oriented programming language that was developed by Sun Microsystems to be network capable. Applications written in Java are compiled into an architecture-neutral object file format that can in principle be understood and executed by all Java interpreters, although they may differ slightly depending on the platform. A Java application or applet is activated by a command issued by a reference mark on the HTML page. The applet then runs self-sufficiently on the respective local host. For obvious security reasons, the applet is not capable of making system calls or addressing ports of the local host. Thus, the applet is memory resident, and data to the remote host can only be transmitted directly via cgi-script (see below). Within this framework, Java creates classes with inheritance, data encapsulation, type casting, and multithreading.
Applets are started whenever the respective HTML page is visited by a Java-capable Web browser. Java contains methods that deliver information about the display monitor (size, number of colors, and resolution) and about the operating system and processor type of the local host computer. Hence, it was possible to tailor the present experimental display to the hardware of the participant. Applets also enable the programmer to handle events - that is, to make actions contingent on events such as mouse clicks or keyboard input. Using event handling, we were able to present equivalent graphical output with the desired spatial layout and timing.

\section{The Experimental Paradigm}

To put the capabilities of Java to a test, the following experiment was designed. It simulated a simple moving target whose motion had to be extrapolated and whose arrival time at a marked location had to be anticipated. A circular object representing a ball moved on a linear path. It disappeared after $1 \mathrm{sec}$ and observers were asked to extrapolate the motion and press a key or click a mouse button when they thought the object had reached a variableposition line. Figure 1 depicts a schematic of the stimulus setup.

The motion trajectory could be parallel to the bottom of the screen, with the ball moving from left to right or bottom to top. Four types of motion were presented: (1) continuous motion, which was approximated by the $72-$ or $60-\mathrm{Hz}$ update rate of the graphics display, depending on the monitor that was used; (2) five-step apparent motion (AM 5), where the target appeared at five successive locations for approximately $40 \mathrm{msec}$ each at intervals corresponding to the continuous case; (3) three-step apparent motion (AM 3), which was the same as AM 5 with the second and fourth blink removed; and finally (4) rabbit motion. Here the object was presented in five flashes, as before, but only in three locations; that is, the whole motion consisted of two double and one single flash, the former recurring at the same location (see Geldard, 1975, for theories about how this stimulus should perceptually distribute; Hecht \& Hoffman, 1999).

The motion interpolation system of humans has been hypothesized to be negatively affected by apparent motion - that is, by time sampling (Elliot, Zuberec, \& Milgram, 1994; Peterken, Brown, \& Bowman, 1991). In this case, time-to-contact judgments should be too long, which indicates an underestimation of velocity.

Half of our participants were randomly assigned to a fixation condition and had to fixate a cross at the top (or right side) of the screen. The final object of investigation was the effect of background on the accuracy of motion extrapolation. Objects in spatial proximity to a moving stimulus (real or apparent motion) are known to interfere with perceived speed (Bowne, McKee, \& Glaser, 1989). Therefore, proximal objects that did not interfere but that suggested a spatial gradient along the motion path should lead to an underestimation of velocity if they induced a 3-D interpretation of the scene. 
In order to reduce the number of stimuli for each participant, the different conditions were mostly run between participants in such a way that each participant saw only two different background conditions (no background and one 2-D or 3-D background), but they saw all flashing types. Also, four extrapolation distances were randomly chosen from trial to trial. Thus, each observer went through 32 trials, which took about $12 \mathrm{~min}$. All betweensubjects conditions were randomly assigned at the outset of the experiment.

\section{Implementation}

As soon as a potential participant visited the experimental website, she/he could choose whether to read the instructions in German or in English. The experimental Web page is accessible under http://www. TechFak. Uni-Bielefeld.DE/techfak/ags/wbski/DSD/blink/ eStart.html

First, a form regarding personal data (age, gender, occupation, and country of residence) had to be filled out. Next, an experimental condition was picked at random, and the appropriate applet was launched. The instructions were presented on a separate screen. Participants were told that a red object (dot) would move across the screen and disappear. The task was to extrapolate the motion and indicate with a mouse click when the ball would have reached the green reference line had it continued its motion. Depending on the condition, the starting positions of the target and the fixation instruction were given. The participant could now press a start button if the experiment appeared interesting, or she/he could directly move to a documentation page.

On start, the applet caused a scene to be drawn onto the participant's monitor using an invisible window that covered the whole display area. The displays were created by combining techniques such as double buffering, waiting for processes to complete, clipping, and buffering (flushing the complete buffers into the visible frame). Thus, we were able to obtain a perceptually smooth animation of the moving disk. The Appendix provides a simplified example of the Java code used to display the stimuli.

Once all trials had been completed (or the experiment had been aborted), the collected data about extrapolation judgments, event timing, and display resolution were emailed to our account. Approximately $95 \%$ of all participants saw animations with the desired time parameters (see below for summary statistics). The following data were collected for each participant.

User information. The name, age, sex, profession, and country of residence of each participant were collected. With the exception of country, this information obviously could not be validated.

Data about the system used. The participant's operating system, resolution and monitor size (width and height in pixels, dots per inch), and information about the browser, including the Java version that was used, and the update rate of the graphics display were identified.
Experimental environment. Time and date of experiment could be directly obtained by accessing the system clock. To ensure that the computer would perform at its capacity, an invisible gauge trial was timed so that the actual run time for a display could be measured. If the display took longer than the $1 \mathrm{sec}$ that it was designed to take, participants were asked to close all other running applications and restart the experiment. To calculate the actual duration of the animation sequence, it was necessary to determine the starting position of the target stimulus and the position where it should have been after $1,000 \mathrm{msec}$. By accessing the system clock of the local host, we could determine the time that had elapsed for the distance that should have been covered within $1,000 \mathrm{msec}$.

Judgment data. For each trial, the contact time between ball and target line as predicted for an ideal participant was compared with the actually produced judgment.

\section{The Challenge of Controlled Data Transfer}

All current operating systems allow multitasking (where multiple processes run in parallel). A resourceconsuming graphics animation is likely to compete with other processes for CPU time. It is thus exceedingly difficult to ascertain a smooth animation at the participant's end. For obvious reasons, the Java script cannot interfere with or prioritize non-Java processes running on the local host computer. Information about the performance of the system on a given experimental trial could in principle have been analyzed by the applet itself or provided remotely. Participants could have been asked to rerun trials that were affected by other processes running on their machine. However, if the applet had rerun substandard trials automatically, we could not have predicted how often a given trial would have been rerun. We opted against this method. We could also have let our server make such a decision, but this would have required sufficient permission for the remote Java applet to write onto our server, which was not the case. Our university's security policy did not allow us to give write privileges. The reverse casefor us to write onto the local host of the participant-was not desirable for obvious reasons, although it would have simplified many things.

The limitation of write permission onto our file system became important in the method we used to collect the data that were produced by the participant. Java could handle a direct data link, but since this was impossible for security reasons, there was only one way to transfer data between the remote host and the experimental site. To our knowledge, the only possibility was a socket connection to the remote host, which enabled data to be sent by means of a cgi-script. The cgi-script executed and sent the data as e-mail.

Java has direct calls that assess the host's screen resolution and its pixel size. Likewise, information about operating system, CPU, and the Web browser that was used can be directly obtained. Unfortunately, however, Java is not capable of controlling or informing about the refresh 
Table 1

Summary Statistics on the First 500 Participants Who Have Started the Experiment

\begin{tabular}{lr}
\hline Characteristic & $\%$ \\
\hline Gender & 70.3 \\
Male & 29.7 \\
Female & \\
Age (in years) & 23.9 \\
$<20$ & 41.4 \\
$20-29$ & 22.5 \\
$30-40$ & 12.1 \\
$>40$ & \\
Location & 45.8 \\
North America & 2.3 \\
Latin America & 44.3 \\
Western Europe & 5.3 \\
Eastern Europe & 2.0 \\
Asia & 0.3 \\
Australia & \\
Resolution of monitor (in pixels) & 16.3 \\
$640 \times 480$ & 36.6 \\
$800 \times 600$ & 27.6 \\
$1,024 \times 768$ & 6.4 \\
$1,152 \times 900$ & 2.0 \\
$1,152 \times 864$ & 8.4 \\
$1,280 \times 1,024$ & 2.6 \\
Other & \\
Operating system & \\
UNIX & \\
Macintosh & \\
Windows & \\
Browser & \\
Netscape & \\
Explorer & \\
Other & \\
& \\
\hline
\end{tabular}

rate of the monitors. Guesses about the refresh rate could be made only indirectly based on the operating system and the resolution. It is, however, safe to assume that the lowest refresh rate was $50 \mathrm{~Hz}$. Of greater concern than the refresh rate is the rate of animation update that was actually achieved. If the CPU was slow or busy with other processes, the whole animation sequence could have been seriously slowed down to point where the display was rendered worthless for our purposes. Fortunately, there was an indirect way to find out whether or not the experimental displays were updated at the desired rate. As noted, we used a gauge trial and accessed the system clock to determine how long a procedure took that should have lasted exactly $1,000 \mathrm{msec}$. This speed check was run before the experimental stimuli were displayed.

\section{Experiences With the \\ Dynamic Perception Experiment}

The experiment went on-line in March 1997. It was advertised on a website that had an index of a large number of psychological studies on the Internet, called the Experimental Psychology Lab (http://www.unituebingen.de/uni/sii/ulf/lab/webexppsylabd.html). Also we registered our site with various WWW search engines such as AltaVista. The main search keywords were experiment, game, visual, perception, Java, and psychol$o g y$ (in both English and in German). In addition, we inserted links to the experiment on our respective home pages. We did not announce the experiment in lecture or seminar classes to avoid sampling large numbers of undergraduate psychology students.

By the end of September, 502 Web users had visited the site and pushed the start button for the experiment. A total of $7.4 \%$ aborted the experiment before the first data point could be collected. Another $18.5 \%$ of participants finished less than the required 32 trials. The remainder completed the experiment. Table 1 provides an overview of the important viewer and system variables that were collected from all participants.

Among the completed data sets, the most critical information to evaluate is the performance of the local host with respect to maintaining a smooth animation of the displayed motion sequence. The analysis of the preexperimental gauge trials revealed that for a desired presentation time of $1,000 \mathrm{msec}$, an average actual presentation time of $1,026.6 \mathrm{msec}$ was obtained. This value was associated with a standard deviation of $233 \mathrm{msec}$. However, the distribution of gauge times was clearly bimodal. While the large majority was within a narrow margin of 1,000 msec, approximately $7 \%$ of the data clearly fell outside that margin, exceeding $1,000 \mathrm{msec}$ by between 200 and $900 \mathrm{msec}$. This has to be attributed to nonoptimal performance of the system. Once these obvious outliers were removed, the remaining errors were well within the accuracy of about $30 \mathrm{msec}$ within which the $1,000-\mathrm{msec}$ gauge interval could be measured. Once the test block had begun, no further system performance checks were made. Such controls consume valuable system time and might have interfered with the displays.

Thus, overall the displays were presented on most local hosts with no or very small delays. The performance data would, however, argue against interpreting timing differences around 10 or $20 \mathrm{msec}$ unless the performance of the local host is known explicitly.

\section{Comparison Between Web and Lab Data}

Further analyses included only the first 54 participants who completed all trials and could not be classified as outliers with respect to the performance of their respective local host computers. This cutoff was made to avoid inflating the statistical analyses.

One way to test the validity of data collected over the WWW is obviously a direct comparison with data obtained in a controlled lab setting. Thus, the experiment was replicated in a controlled laboratory setting. Since it was prohibitive to collect between-subjects data for all conditions, only one background (without features) was used. The stimuli were displayed on a Silicon Graphics Indigo $2 \mathrm{XZ}$ workstation with a resolution of $1,280 \times$ 1,024 pixels and a refresh rate of $72 \mathrm{~Hz}$ (noninterlaced). The animation update rate was also 72 frames/second. 
Table 2

Comparison Between Data Collected in the Lab and Via the Internet in Milliseconds

\begin{tabular}{lrccc}
\hline & \multicolumn{4}{c}{ Error Type } \\
\cline { 2 - 5 } Motion Type & CE & $S D$ of CE & AE & $S D$ of CE \\
\hline \multicolumn{5}{c}{ Web Experiment } \\
C & 33.6 & 203.5 & 136.7 & 154.2 \\
AM 5 & 96.7 & 188.7 & 147.4 & 152.3 \\
AM 3 & 171.3 & 197.6 & 195.4 & 173.7 \\
R & 345.2 & 232.5 & 357.3 & 213.4 \\
& \multicolumn{5}{c}{ Lab Experiment } \\
C & 92.5 & 197.2 & 159.3 & 148.5 \\
AM 5 & 144.8 & 215.7 & 200.6 & 165.1 \\
AM 3 & 163.6 & 260.3 & 242.2 & 189.2 \\
R & 164.8 & 234.6 & 224.4 & 178.4 \\
\hline
\end{tabular}

Note Observers tended to be late in their reaction, as indicated by the positive numbers. In both cases, data are averages for each of the four motion conditions (continuous motion [C], five-step apparent motion [AM 5], three-step apparent motion [AM 3], and rabbit motion [R]). $\mathrm{CE}$, constant error; $\mathrm{AE}$, absolute error; $S D$, standard deviation.

Eight male observers were paid for their participation. The observer was seated $52 \mathrm{~cm}$ away from the 20 -in. monitor, with his line of sight at the center of the display screen. Details of the lab experiment can be found in Hecht and Hoffman (1999). After the data had been collected, obvious outliers were removed from the analysis. When the observer responded within the presentation time and before the extrapolation period had started, an accidental mouse click was assumed. Likewise, when the reaction was more than $1,000 \mathrm{msec}$ after true time to contact with the reference line, a missed trial was assumed. According to this criterion, $1 \%$ of all lab trials and $13.7 \%$ of all Web trials were removed from the analysis.

As shown in Table 2, when analyzed as a function of motion type, the data collected via the Web followed the same pattern as those produced under laboratory settings. Continuous motion was easiest to extrapolate; AM 3 led to larger underestimation of velocity than did the AM 5 . A regression analysis based on the cell means-that is, the constant error values for all four motion conditionswas performed. The timing errors correlated highly between Web and laboratory studies $(r=.77)$. This correlation is not as impressive as those obtained by Krantz, Ballard, and Scher (1997), which approached unity. They conducted a comparison between Web and lab data based on ratings of stationary images by a large number of observers. Thus, as one might expect, the dynamic stimulus presentation is inferior to stationary images. Our justmentioned regression analysis did not reveal significant differences between Web and lab timing data $[F(1,4)=$ $2.93, p=.23$ ]. However, this analysis was based on averages and is likely to have hidden smaller differences. When one is evaluating the differences between two studies, such as the Web and the laboratory experiment, what constitutes a conservative measure is not defined.

Consequently, additional repeated measures analyses of variance (ANOVAs) were run separately for the raw Web and lab data. One difference that was found in this analysis relates to the rabbit motion. Rabbit motion was not significantly different from AM 3 under lab conditions, but Web participants produced larger errors when confronted with rabbit displays $[F(1,49)=277.14, p<.0001]$. Several factors might be responsible for this deviation. First, the resolution of the lab monitor was well above average. Only four Web participants had 1,280 × 1,024 displays. Second, some computers used by Internet participants may not have been able to update their displays at the $72-\mathrm{Hz}$ refresh rate that was used in the laboratory.

Another difference between Web and lab data was found for the fixation instruction. Within the laboratory setting, the instruction to track the moving dot produced larger estimation errors of contact time than did the instruction to fixate a stationary cross $[F(1,28)=5.30, p=$ $.0289]$. Thus, peripheral vision facilitated the process of motion interpolation. Such an effect was absent in the data collected via the WWW. Presumably some participants did not read, understand, or obey the fixation instruction. The background manipulation was unique to the Web experiment. Unfortunately, a repeated measures ANOVA revealed no significant differences in time to contact judgments as a function of background, but a trend for the 3-D background to lead to later responses emerged.

In sum, the results gathered from Web participants were very promising and should encourage use of this method of data collection in other domains as well. However, several caveats should be noted. First, hardware and resource allocation on the local host machine were often not up to laboratory standards. As a consequence, resolution and frame rate suffered. These technical problems seem surmountable. Data collected on substandard systems can easily be excluded from the data set. Moreover, given the fast progress in hardware development, technical problems will become less troublesome in the future.

Second, and more seriously, the Web experiment involved a lack of experimental control. We do not know what the lighting conditions, viewing position, and visual viewing angles were at the sites of the Web experiments, nor do we have information about level of attention, noise, other distractions, and so on. These cannot easily be measured or reported. Moreover, the viewing environment may have varied considerably between participants, especially considering that they were spread across the globe. Given all these problems, it is rather encouraging that the data were very close to those collected in a controlled laboratory environment. The fact that the fixation instruction produced no effect, however, is troublesome. Observers may not have read the instructions carefully enough, may just not have cared, or may have deliberately ignored instructions. At first sight, as experimenters we have no way of telling whether the effect is real or attributable to instructions that were not followed. Catch trials or special questions to detect liars will obviously not cure the problem.

In our opinion, this challenge to experimental control encountered in WWW experiments is qualitatively the same as in the laboratory, albeit quantitatively different. In either case, we need to rely on participants who 
are willing to cooperate and able to understand instructions. And in both cases the experimenter - according to her/his subjective criteria-decides whether or not the participant has conformed to the experimental task and been a good participant or whether the data are not usable. It seems to hold that one must demand less of a Web participant; that is, the more critical and refined the viewing instructions, the less suitable the experiment for porting onto the WWW.

Given the experimental control problem associated with WWW experiments, what are the realistic advantages of doing dynamic Internet experiments? First, displays programmed in Java are a powerful tool to create platform independent experiments. The same program can be run in different labs. It is thus possible to collect data for the same experiment in different locations. This is particularly helpful when different researchers collaborate across labs. We have, for instance, programmed another experiment where the development computer and the lab computer used for data collection are in different buildings on the same campus. Moreover, the experiment can be shown all over the world for demonstration purposes without the necessity of carrying around disks or transferring files and installing the experimental software at the remote site.

Experiments implemented on the WWW are also a powerful resource for collecting pilot data, in particular when large populations need to be subjected to few trials. Once a link to our Web experiment was established at a server that refers to and briefly describes all psychological Web experiments in Germany (http://www.unituebingen.de/uni/sii/Ulf/Lab/WebExpPsyLabD.html), data came in at very fast rates. Similar sites in the United States could also provide fast pointers to psychological experiments (see the above-mentioned link to the NASA Vision Science page, which provides links to many areas of visual perception).

\section{Conclusion}

The present results are encouraging; the perceptual motion extrapolation experiment that was implemented using Java yielded results that confirm some important effects that were obtained in a laboratory setting. Taking into account the comparatively loose control of the experimental situation via the WWW, the Web data provide evidence for the robustness of the time-sampling effect. At the same time, clear limits of the method were found in how far participants seemed to follow instructions, such as the fixation instruction. Given the continuously improving hardware and software, the lack of control over the participant's viewing position, alertness, cooperativeness, and so on, appears to be the one serious shortcoming of Web-based data collection. This lack of experimental control has some practical implications for running perception experiments on the WWW. Obviously, the casual environment of the typical net browsing participant will never do justice to the many laboratory requirements. The lighting in the room, interruptions, care of reading the instructions, as well as the focus of attention are likely to vary considerably between net participants. Thus, only those studies that can afford relaxed viewing requirements lend themselves to the type of experiment that we have implemented. These will be mostly pilot studies. Also, consider that the timing study that we chose tested the limits of such Web applications; other dynamic studies that are not as time critical are likely to yield even better congruence between Web and lab data.

However, the use of the WWW goes far beyond the collection of pilot data. Java permits the experimenter to run one and the same experiment anywhere in the world. It is thus conceivable that several experimenters in different locations share one experimental setup. Once an experimenter is present, she/he can check laboratory standards while the experiment is running via the Internet. A mobile experimenter is conceivable; rather than moving the lab equipment, he/she could always have access to all necessary experimental devices through the Internet. We foresee particular benefits whenever large subject numbers are required and in the context of individual differences such as those explored in cross-cultural studies.

\section{REFERENCES}

Bailey, E. K., \& Cotlar, M. (1994). Teaching via the internet. Communication Education, 43, 184-193.

Bowne, S. F., McKeE, S. P., \& Glaser, D. A. (1989). Motion interference in speed discrimination. Journal of the Optical Society of America $A, 6,1112-1121$.

Elliot, D., Zuberec, S., \& Milgram, P. (1994). The effects of periodic visual occlusion on ball catching. Journal of Motor Behavior, 26, 113-122.

Flanagan, D. (1996). Java in a nutshell. Sebastopol, CA: O'Reilly. GeldaRd, F. A. (1975). Sensory saltation: Metastability in the perceptal world. Hillsdale, NJ: Erlbaum.

Hecht, H., \& Hoffman, D. D. (1999). Extrapolating visual motion: Effects of spatial and temporal sampling. Manuscript submitted for publication.

Hewson, C. M., Laurent, D., \& Vogel, C. M. (1996). Proper methodologies for psychological and sociological studies conducted via the Internet. Behavior Research Methods. Instruments, \& Computers, 28, 186-191.

Kelley-Milburn, D., \& Milburn, M. A. (1995). Cyberpsych: Resources for psychologists on the Internet. Psychological Science, 6, 203-211

Kent, P., \& Kent, J. (1996). Official Netscape Java Script book: The nonprogrammer's guide to interactive Web pages. Research Triangle Park, NC: Ventana Communications.

Krantz, J. H., Ballard, J., \& Scher, J. (1997). Comparing the results of laboratory and World-Wide Web samples on the determinants of female attractiveness. Behavior Research Methods, Instruments, \& Computers, 29, 264-269.

Peterken, C., Brown, B., \& Bowman, K. (1991). Predicting the future position of a moving target. Perception, 20, 5-16.

Trapp, A., Hammond, N., \& Bray, D. (1996). Internet and the support of psychology education. Behavior Research Methods, Instruments. \& Computers, 28, 174-176.

Welch, N., \& Krantz, J. H. (1996). The World-Wide Web as a medium for psychoacoustical demonstrations and experiments: Experience and results. Behavior Research Methods, Instruments, \& Computers, 28, 192-196. 


\section{APPENDIX}

This shortened part of the Java source shows the flow of control. The source code is in boldface; the comments are in plain text.

1. Defining variables

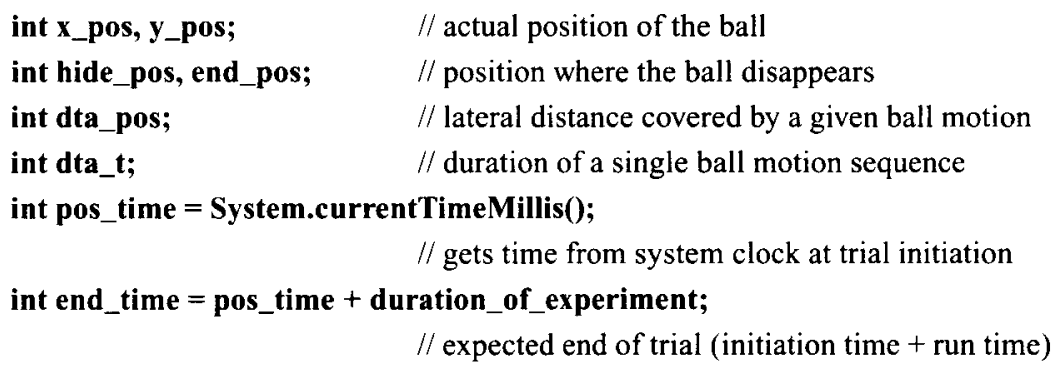

2. The loop creating smooth movement from top to bottom:

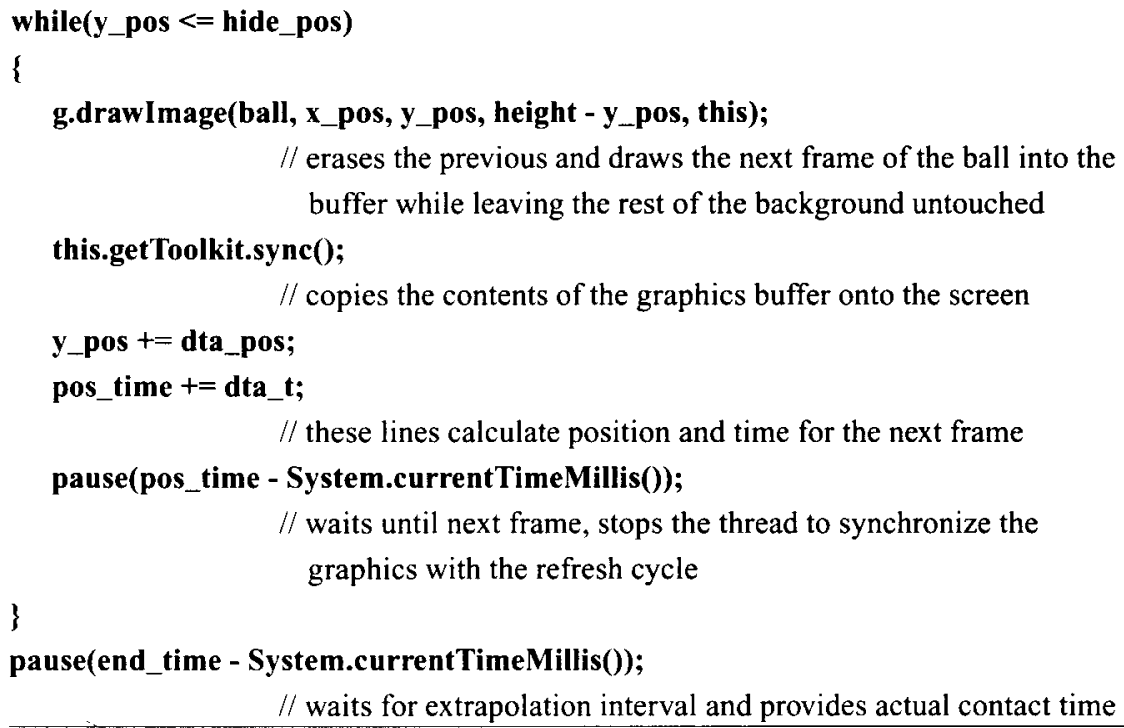

